\title{
Technology Roadmapping, um método para apoiar a gestão tecnológica
}

\section{Silvia Satiko Onoyama Mori}

Doutoranda em Administração pelo Programa de Pós-Graduação em Administração (PPGA) da Universidade de Brasília (UnB). Pesquisadora da Empresa Brasileira de Pesquisa Agropecuária (Embrapa). Membro do grupo de pesquisa Socie-dados da UnB, São Paulo, Brasil

silonoyama@gmail.com/silvia.onoyama@embrapa.br

Eduardo Machado Cruz

Doutor em Genética e Melhoramento pela Universidade Federal de Viçosa (UFV). Analista da Empresa Brasileira de Pesquisa Agropecuária (Embrapa), São Paulo, Brasil

eduardo.cruz@embrapa.br

Alice Kazuko Inoue Nagata

Doutora em Fitopatologia pela Universidade de Brasília (UnB) com desenvolvimento da tese na modalidade "sanduíche" na Wagningen University, Holanda. Pesquisadora da Empresa Brasileira de Pesquisa Agropecuária (Embrapa), São Paulo, Brasil

alice.nagata@embrapa.br

Jonathan Simões Freitas

Doutor em Administração pelo Centro de Pós-Graduação e Pesquisas em Administração (CEPEAD) da Universidade Federal de Minas Gerais (UFMG). Pesquisador do Núcleo de Tecnologia da Qualidade e da Inovação (NTQI/DEP) e do Núcleo de Ensino e Pesquisa em Mercadologia e Estratégia em Organizações (NUME/DCA) da UFMG. Gerente geral e coordenador de pesquisa de um projeto transdisciplinar da Associação Kuyper para Estudos Transdisciplinares, financiado pela Templeton World Charity Foundation, Minas Gerais, Brasil jonathan.ufmg@gmail.com

Editor Científico: José Edson Lara
Organização Comitê Científico
Double Blind Review pelo SEER/OJS
Recebido em 09.07.2016
Aprovado em 28.06.2017

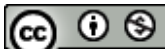

Este trabalho foi licenciado com uma Licença Creative Commons - Atribuição - Não Comercial 3.0 Brasil
} 


\section{Resumo}

Esta pesquisa teve como objetivo aplicar o método Technology Roadmapping (TRM) para o planejamento tecnológico de pesquisa em tomateiro de mesa, com foco em fitossanidade (doenças e pragas). A pesquisa-ação foi utilizada para o desenvolvimento do estudo obedecendo às seguintes etapas: (1) reflexão inicial exploratória e definição do problema e do escopo; (2) planejamento da pesquisa, com a realização de pesquisas em bases primárias e secundárias para levantamento de informações de mercado, do produto e de tecnologias; (3) execução das ações estabelecidas, com a construção do Roadmapping sobre pragas e doenças do tomateiro; (4) realização de análise das observações, reflexões e avaliações, que ocorreram durante o processo de pesquisa. Como resultado, a aplicação do TRM permitiu uma proposição clara de ações voltadas a atender as principais demandas relacionadas à fitossanidade para tomateiro de mesa.

Palavras-chave: Prospecção tecnológica; planejamento tecnológico; roadmapping; fitossanidade.

\section{Technology Roadmapping, a method to support technology management}

\section{Abstract}

The objective of this study was to apply the Technology Roadmapping (TRM) method for technologically planning the research on tomatoes for fresh market, with the focus in phytosanitary (diseases and pests) problems. The research-action was employed based on the following steps: (1) initial exploratory reflection and definition of the problem and the scope; (2) research planning, by performing research in primary and secondary basis to assess information regarding the market, the products, and the available technologies; (3) performing the actions that were established by the construction of the Roadmapping on diseases and pests of tomato plants; (4) analyses of the observations, reflections and evaluations done during the entire process. The application of the TRM method resulted in a clear proposition of actions devoted to meet the major demands related to the phytosanitary issues on fresh market tomatoes.

Keywords: Technological prospection; technological planning; roadmapping; plant health.

\section{Technology Roadmapping, un método para apoyar la gestión tecnológica}

\section{Resumén}

Este estudio tuvo como objetivo aplicar el método Tecnología Roadmapping (TRM) para planificación de la investigación tecnológica en el tomate in natura, centrándose en la sanidad de las plantas (enfermedades y plagas). Se utilizó la investigación de acción para el desarrollo del trabajo, obedeciendo las siguientes etapas: (1) la 
reflexión exploratoria inicial en la definición del problema y el alcance; (2) búsqueda de planificación para llevar a cabo la investigación en el almacenamiento primario y secundario para levantamiento de información de mercado, productos y tecnologías; (3) la ejecución de las acciones establecidas con la construcción de Roadmapping sobre plagas y enfermedades del tomate; (4) observaciones, reflexiones y evaluaciones se produjeron durante el proceso. Como resultado, la aplicación de TRM permitió una clara proposición de medidas para abordar las demandas clave relacionadas con de cultivo de tomate para consumo.

Palabras clave: Tecnología de previsión; planificación de la tecnologia; roadmapping; sanidade vegetal.

\section{Introdução}

A inovação, em termos de novos produtos, processos e serviços, é um componente crítico para que empresas possam criar valores e manter sua competitividade (Subramaniam \& Youndt, 2005). Esse processo requer um melhor entendimento das relações entre o ambiente externo, as capacidades tecnológicas e os objetivos corporativos em face da rápida mudança tecnológica (Carvalho, Fleury \& Lopes, 2013; Phaal, Farrukh \& Probert, 2004; Probert \& Radnor 2003).

Nas Instituições de Ciência e Tecnologia (ICTs) brasileiras, essa necessidade é pautada por uma demanda crescente do governo, dos investidores e da sociedade e por avaliações de resultados nas organizações que, tradicionalmente, investem em pesquisa e desenvolvimento (Marques, Vedovoto \& Avila, 2009). No âmbito da Embrapa, há um esforço da organização para que sejam incorporadas, de forma sistemática, as demandas tecnológicas identificadas das cadeias produtivas nos projetos de Pesquisa e Desenvolvimento (P\&D), com vistas a consolidar o processo de inovação (Empresa Brasileira de Pesquisa Agropecuária [Embrapa], 2015). Isso faz com que sejam aumentadas as chances de as instituições de P\&D, como a Embrapa, garantirem sua importância, legitimidade e sustentabilidade (Bassi, Silva, Leis \& Poit, 2015).

Sendo assim, a utilização de métodos e ferramentas que auxiliem no planejamento tecnológico se torna cada vez mais necessária para o processo de inovação (Coelho, Santos, Santos \& Fellows Filho, 2010). Um dos métodos que tem proporcionado essa interlocução é o Technology Roadmapping (TRM) ou Roadmapping (Carvalho et al., 2013; Phaal et al., 2004; Probert \& Radnor 2003). A 
adaptabilidade do TRM a situações e objetivos distintos tem possibilitado sua aplicação tanto em estudos de ciência e tecnologia como em setores industriais específicos (Lee, Phaal \& Lee, 2011).

Diante do exposto, este estudo tem como objetivo mostrar a aplicação do método TRM para auxiliar no direcionamento da pesquisa na cadeia produtiva do tomate de mesa, em um horizonte de longo prazo, considerado como sendo de 10 anos. A cadeia do tomate é uma das principais cadeias produtivas de hortaliças (Instituto Brasileiro de Geografia e Estatística [IBGE], 2015), sendo o foco deste estudo a fitossanidade (doenças e pragas), pelo fato de representar um dos principais gargalos na produção, considerando a perspectiva dos agentes da cadeia, devendo sempre haver monitoração de forma que se possam minimizar problemas existentes e se antecipar a possíveis novas pragas e doenças. Essa iniciativa faz parte do fortalecimento dos processos de planejamento tecnológico das cadeias produtivas olerícolas.

\section{Referencial Teórico}

O TRM ou Roadmapping emergiu como um método flexível, sendo amplamente utilizado na indústria e em políticas setoriais para apoiar o planejamento estratégico das organizações ou do setor (Carvalho et al., 2013; Kerr \& Phaal, 2015; Phaal, Farrukh, Mitchell \& Probert, 2003; Phaal, Farrukh \& Probert, 2010). A abordagem gráfica do TRM permite explorar e comunicar as relações entre evolução e desenvolvimento de mercados, produtos e tecnologias, ao longo do tempo (Kerr \& Phaal, 2015; Phaal et al., 2003), além de possibilitar elencar as capacidades tecnológicas e recursos necessários (Phaal et al., 2004).

Desse modo, é possível alinhar as diversas funções e perspectivas dentro de uma organização e fornecer um quadro estruturado para responder a três questões fundamentais: Onde estamos agora? Para onde queremos ir? Como poderemos chegar lá? (Phaal \& Muller, 2009). Nesse sentido, o TRM apoia a organização na tomada de decisão quanto aos investimentos, além de aperfeiçoar a coordenação de esforços para que os objetivos sejam alcançados.

Kappel (2001) e Schuh et al. (2011) detalham que esse método contribui para a compreensão do ambiente competitivo por meio das informações advindas do benchmarking com os concorrentes e do monitoramento de ambiente externo para o alinhamento de objetivos e estratégias. O TRM auxilia na priorização de projetos de 
P\&D, considerando as informações acima levantadas (Kappel, 2001; Oliveira \& Rozenfeld, 2010). Ademais, esse método contribui para a sincronização do planejamento estratégico da empresa com a gestão da carteira de projetos. Além disso, o uso desse método melhora a transparência do planejamento, oferecendo subsídios para o corpo gestor agir em situações complexas (Schuh et al., 2011).

A arquitetura básica do TRM, segundo Phaal, Farrukh e Probert (2001) e Phaal et al. (2003), Probert e Radnor (2003), é uma representação gráfica, ou roadmap, formada pela linha de tempo do eixo horizontal e por três camadas no eixo vertical, as quais representam as dimensões de mercado, produto e tecnologia (Figura 1). A primeira camada contempla a compreensão do ambiente competitivo em que a organização está situada e o planejamento de novos cenários. A segunda camada remete ao planejamento dos projetos de produtos, dos processos e dos serviços, levando em conta o cenário levantado. A terceira camada permite a identificação de novas tecnologias que auxiliarão no desenvolvimento do portfólio de produtos, processos e serviços.

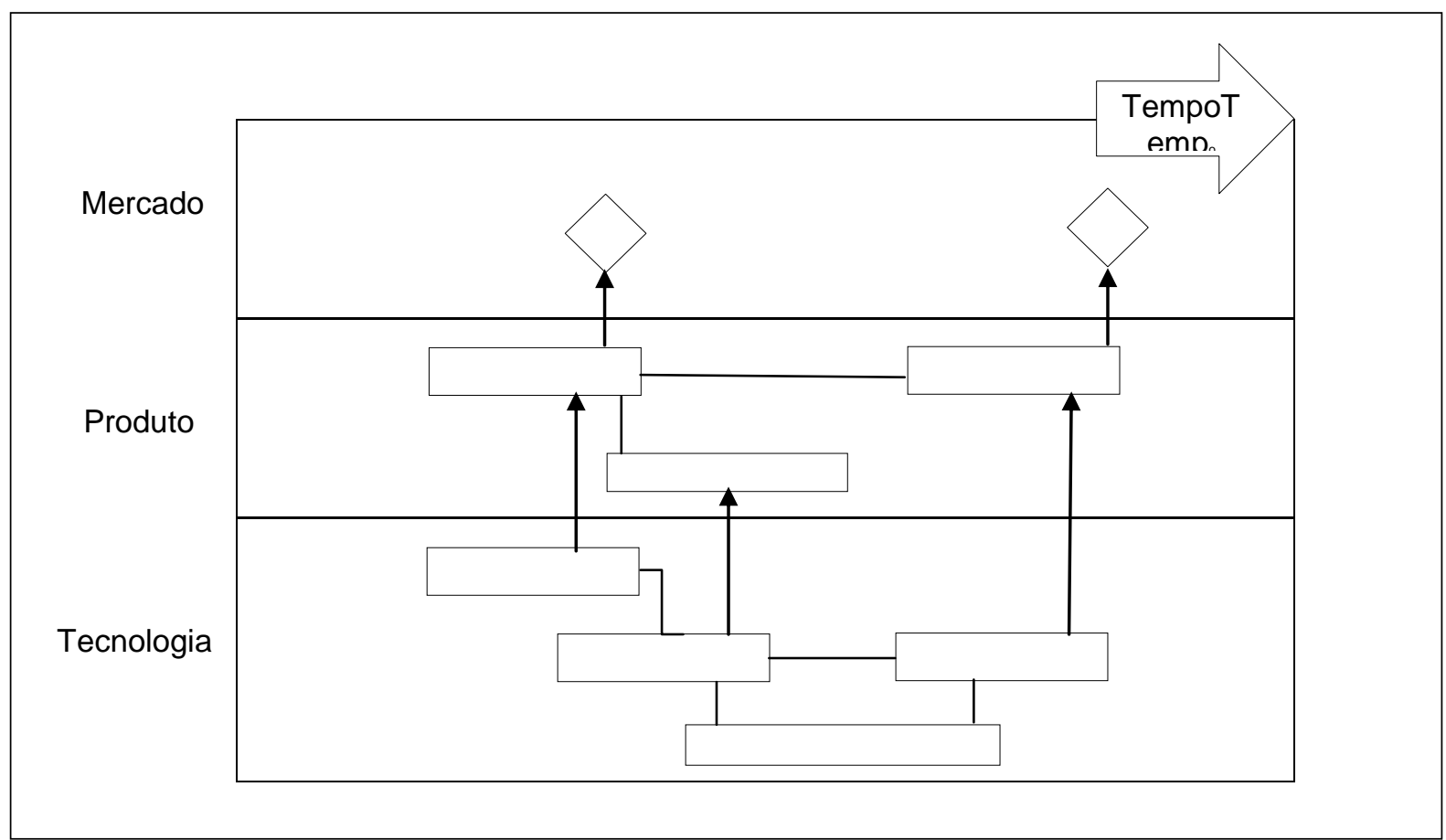

Figura 1 - Arquitetura básica do TRM

Fonte: Phaal, R., Farrukh, C., Mitchell, R., \& Probert, D. (2001). T-Plan: Fast Start to Technology Roadmapping Planning Your Route to Success. University of Cambridge; Phaal, R., Farrukh, C., Mitchell, R., \& Probert, D. (2003). Starting-up roadmapping fast. Research-Technology Management, $46(2), 52-59$. 
A estrutura básica do TRM pode ser desdobrada em vários modelos e tipos de rotas tecnológicas, conforme os propósitos de cada organização (Lee et al., 2011; Phaal et al., 2004). As estruturas podem englobar essas três camadas, ou um número maior de camadas para uma abordagem mais estratégica, ou ainda ser mais específica para a gestão de produtos e tecnologias (Oliveira et al., 2012). Essa flexibilidade, que permite a customização da arquitetura básica do TRM, é muito importante para atender às necessidades específicas dos usuários (Lee \& Park, 2005; Oliveira et al., 2012).

Em seu estudo sobre o planejamento da ciência e tecnologia na China, Huang, Zhang, Guo, Zhu e Porter (2014) customizaram o TRM em quatro dimensões: nação; tecnologia; indústria; riscos e impacto. Os autores procuraram mapear o contexto de tecnologias-chave para o desenvolvimento da nação em questão em termos desdobramento tecnológico, setores industriais que utilizam as tecnologias-chave e potenciais impactos das inovações a serem geradas com as tecnologias portadoras do futuro.

No caso do planejamento estratégico, o roadmapping pode ser customizado para atender às necessidades das organizações. Como exemplo de um TRM no âmbito de uma instituição de pesquisa, Loyarte et al. (2015) desenvolveram um TRM para o planejamento estratégico que englobou projetos a serem conduzidos, protótipos a serem desenvolvidos, linhas de pesquisas e recursos necessários em face das tendências mapeadas previamente do ambiente externo.

Quando o objetivo é duscutir o portfólio de produtos e tecnologias, somente as duas últimas camadas são utilizadas para a elaboração dos roadmaps (Lee \& Park, 2005). Nessa direção, os autores elaboraram um conjunto de roadmaps para discutir família de produtos e de tecnologias que englobou mapeamento da família de produtos e de tecnologias, roadmap do planejamento do produto e das tecnologias prospectadas, direcionadores de produto e de tecnologia, evolução do produto e tendências tecnológicas.

Independentemente da arquitetura adotada, a aplicação do TRM voltada para a gestão tecnológica obedece à lógica do processo de implantação proposto por Phaal et al. (2004), que consiste nos seguintes passos: seminário de planejamento; seminário de mercado/negócio; seminário de produto; seminário de tecnologia; e seminário de mapeamento da rota tecnológica. Os seminários são fundamentais para a elaboração do TRM, uma vez que fornecem espaço para reunir pessoas dos 
diversos setores da organização (Phaal \& Muller, 2009) com o intuito de estimular a aprendizagem organizacional sobre 0 processo de planejamento tecnológico (Groenveld, 2007). Além disso, os seminários melhoram a comunicação dos principais stakeholders em relação à programação de P\&D (Grossman, 2004).

Segundo Probert e Radnor (2003), nas etapas de levantamento das informações para os seminários de mercado, produto e tecnologia, o TRM é assessorado por outras ferramentas na construção da rota tecnológica. Essas ferramentas de gestão podem potencializar o TRM, diminuindo as lacunas de conhecimento e auxiliando as tomadas de decisão (Carvalho et al., 2013; Phaal, Farrukh \& Probert, 2005).

No Brasil, há relatos positivos da aplicação do TRM para auxiliar o planejamento tecnológico em alguns setores, sendo alguns deles descritos ao seguir. No nível estratégico, Melo Filho, Gonçalves, Cheng e Muniz (2015) utilizaram a abordagem estratégica de roadmapping na geração de diretrizes de inovação com o propósito de redirecionar um conjunto de firmas localizadas em um conglomerado industrial para atender a um novo setor-cliente com maiores exigências tecnológicas que os setores-clientes usuais.

Considerando o nível de produto, Alves, Silva e Mello (2011) estudaram o emprego do TRM para a seleção de um produto de referência na aplicação da engenharia reversa como parte do processo de desenvolvimento de produtos de uma empresa de base tecnológica (EBT). O estudo teve como resultado o desenvolvimento de um produto que atende às necessidades dos clientes e do mercado, com tecnologias e funcionalidades avançadas e melhoradas.

Já no setor agropecuário, o emprego do TRM no planejamento tecnológico de pesquisa da cadeia produtiva da cenoura mostrou novas tendências e possibilitou o redirecionamento do programa de melhoramento, ampliando as possibilidades da atuação da Embrapa Hortaliças no desenvolvimento de cultivares, considerando um horizonte de 15 anos (Onoyama et al., 2012). No caso do TRM aplicado à cadeia produtiva da cenoura, optou-se por modificar a estrutura original em quatro macrocamadas: mercado, metas de negócio, linhas de pesquisa e recursos.

Natalense e Zouain (2013) propuseram o uso de roadmapping como uma técnica para o planejamento estratégico de longo prazo do desenvolvimento do biobutanol, alinhando objetivos de longo prazo com os recursos, financiamento e 
prioridades, com vistas a atender às necessidades do processo de desenvolvimento no Brasil. Para tanto, os autores utilizaram a arquitetura original do método para a elaboração da rota tecnológica.

Roadmaps bem elaborados permitem aos gestores $\mathrm{e}$ aos técnicos visualizarem os dados no contexto, identificarem padrões e conexões e destacarem questões críticas (Kerr \& Phaal, 2015). O importante é que o roadmap explicite bem o planejamento tecnológico no contexto observado (Oliveira et al., 2012).

\section{Metodologia}

A ordenação metodológica se consistiu em uma pesquisa-ação, uma vez que envolveu os pesquisadores e os membros de uma organização no trabalho de análise de um assunto de genuíno interesse dos participantes em agir com base na intervenção e mudanças propostas (Eden \& Huxman, 2001; Thiollent, 2009). Foram utilizados os preceitos de Martins e Theóphilo (2009), associados às etapas preconizadas por Phaal et al. $(2001,2004)$ e Kerr e Phaal (2015), para estabelecer a sequência da pesquisa, conforme descrito a seguir:

- A reflexão inicial exploratória teve como objetivo o entendimento do problema da pesquisa e a definição da unidade de análise, do escopo, da estrutura do roadmap e dos números de camadas, do horizonte de planejamento e cronograma de atividades. Além disso, para melhorar a operacionalização do trabalho, houve treinamento da equipe sobre o método TRM;

- $\quad$ O planejamento ou pesquisa aprofundada estabeleceu um cronograma das atividades de trabalho. Foram consideradas as seguintes informações para a realização da pesquisa: a) informações econômicas obtidas em levantamentos realizados pela FAO e pelo IBGE; b) informações das cultivares de tomateiro nas homepages das principais companhias comercializadoras de sementes; c) levantamento de patentes relacionadas à cadeia de tomate das principais empresas de semente no Web of Science; d) levantamento dos projetos realizados e em andamento pela Embrapa; e) pesquisa com 38 representantes da cadeia de tomate de mesa para levantar as principais tendências, oportunidades e ameaças do setor. 
Tomando como base as informações levantadas, o objeto do roadmap foi redefinido para doenças e pragas e, com isso, a arquitetura do método TRM também foi redefinida.

- A etapa das ações. Nessa etapa, foram executadas as seguintes ações: i) preparação de um workshop para a construção do roadmap; ii) realização de seminários sobre as camadas do TRM e discussão inicial do roadmap do tomate em um único workshop; iii) reuniões posteriores com os colaboradores internos para complementação do roadmap;

- As observações, reflexões e avaliações ocorreram em várias etapas do trabalho. Houve reuniões gerais, com a equipe interna de fitossanidade da Embrapa Hortaliças, para discussão do andamento das atividades e para delinear o foco do seminário. Ao final, realizou-se um planejamento de ações de pesquisa de curto, médio e longo prazo para a área de fitossanidade de tomate de mesa. Além disso, houve uma reflexão sobre a condução da pesquisa envolvendo a cultura do tomate de mesa, com levantamento de novos pontos positivos e negativos, considerando-se o trabalho realizado.

O trabalho de intervenção foi realizado por um grupo gestor composto pelo Chefe de P\&D da Embrapa Hortaliças juntamente com um analista de pesquisa, desenvolvimento e inovação, além de duas pesquisadoras, uma da área de negócios tecnológicos e outra da área de fitossanidade. Contou-se também com parceiros da Universidade Federal de Minas Gerais que foram os facilitadores da intervenção. O processo de intervenção ocorreu durante os anos de 2013 a 2015. Para realização do workshop e das reuniões, contou-se com a participação de nove pesquisadores que trabalham com a cadeia de tomate de mesa.

\section{Apresentação e Análise dos Resultados}

\subsection{Caracterização da Embrapa Hortaliças}

A Embrapa Hortaliças é uma das 46 unidades descentralizadas e uma das 14 unidades de produtos da Empresa Brasileira de Pesquisa Agropecuária - Embrapa. Ocupando uma área total de 1.204 hectares, onde estão instalados os campos 
experimentais, prédios administrativos, laboratórios, casas de vegetação e estufas e telados, além dos prédios de apoio, a Embrapa Hortaliças tem um total de $22.000 \mathrm{~m}^{2}$ de área construída.

Atuando em Pesquisa, Desenvolvimento e Inovação para a produção sustentável de hortaliças, a Embrapa Hortaliças visa à eficiência e à competitividade desse setor do agronegócio. Referência no Brasil e no exterior, a Embrapa Hortaliças se destaca pela sua contribuição técnico-científica e pela capacidade de articulação nacional e internacional em prol da sustentabilidade da olericultura e do ambiente rural. Além disso, essa unidade realiza parcerias na geração e na transferência de tecnologias para diferentes segmentos sociais, visando a garantir avanços em novas fronteiras do conhecimento e oferecer produtos e serviços, preservando e valorizando a biodiversidade e os recursos naturais.

\subsection{Caracterização do Setor de Tomate de mesa}

O tomate possui grande relevância no cenário internacional e nacional, destacando-se pela alta produção e por seu valor nutricional, sendo uma das hortaliças mais consumidas. No Brasil, o plantio de 2016 abrangeu a área de 58.548 hectares, com a produção de 3.737 .925 toneladas (IBGE, 2017). O tomate figura entre as principais hortaliças do Brasil e o país está entre os dez maiores produtores do mundo (Food and Agriculture Organization of the United Nations [Faostat], 2014).

Diferentemente do tomate para processamento industrial, que é produzido em grande parte com contratos de venda já fechados, a cadeia de comercialização do tomate de mesa, em geral, ainda é muito desorganizada, sendo composta basicamente por três agentes, quais sejam: produtor, atacadista e varejista. A existência do intermediário varia conforme a região produtora.

Segundo a Hortifruti Brasil (2016), o custo de produção do tomate é um dos maiores em toda a atividade agrícola e, em algumas regiões, já ultrapassou $R \$ 100$ mil por hectare. Além disso, o preço e, consequentemente, o retorno econômico, dependem fortemente do aspecto visual do tomate, que é altamente sensível às pragas, doenças, bem como do manuseio e da oferta. A variação de preços está diretamente relacionada à ausência de zoneamento da produção, que impacta a oferta, uma vez que não existe a regulação da disponibilidade com a demanda. 


\subsection{Processo de Intervenção}

O escopo da intervenção, neste estudo, tomou como base toda a programação de pesquisa em tomateiro de mesa da Embrapa Hortaliças. Inicialmente, realizou-se uma primeira reunião com o intuito de discutir o status quo da pesquisa em tomateiro na Unidade estudada, a visão de futuro na perspectiva dos pesquisadores e os procedimentos para alcançar as metas com base nos preceitos de Phall \& Muller (2009).

Em um segundo momento, ficou estabelecida a necessidade do entendimento do contexto da cadeia de valor do tomate de mesa para facilitar o processo de adaptação do TRM. Neste estudo, foram mapeados os elos da cadeia, que representam desde a etapa do plantio até a compra dos produtos, in natura ou beneficiados, pelo consumidor final.

Em seguida, realizou-se a pesquisa bibliográfica para levantamento de dados socioeconômicos e das principais cultivares de tomate de mesa disponíveis no mercado. Também foi realizado um levantamento com 38 agentes dos diferentes segmentos da cadeia de tomate de mesa quanto às principais tendências, oportunidades e ameaças da cadeia, perspectivas de novos produtos, e ferramentas tecnológicas para o setor. Para complementação das informações, realizou-se um levantamento dos estudos já realizados pela Embrapa sobre a cultura do tomateiro de mesa. Além disso, pesquisou-se sobre as patentes das principais empresas das áreas de melhoramento e de insumos para verificar os avanços tecnológicos do setor.

Com base nos levantamentos de dados primários e secundários, o escopo do roadmap foi redefinido, optando-se por realizar o TRM apenas para o tema referente a doenças e pragas, uma vez que esse é o principal problema na área de pesquisa pela ótica dos agentes da cadeia. A complexidade desse tema é alta e, para ser entendida, demanda ações de várias áreas de conhecimento dentro da pesquisa agrícola, envolvendo melhoramento genético, manejo integrado de pragas e doenças, além de processos e cuidados de pós-colheita.

Nesse sentido, a estrutura do roadmap foi modificada em relação à estrutura original proposta por Phaal et al. (2001). Para melhor atender ao objetivo proposto, optou-se por trabalhar as camadas produto e tecnologia, seguindo os preceitos de 
Lee e Park (2005) e Oliveira et al. (2012), tendo como norte as principais doenças e pragas da cultura de tomate de mesa (Figura 2). A camada produto foi trabalhada como metas (resultados) e ações (atividades de pesquisa que serão realizadas), e a camada tecnologia, como recursos dos quais se deve dispor para atender às necessidades estratégicas. A Figura 2 exemplifica o detalhamento de uma doença do tomateiro, a Ralstonia.

A estruturação das camadas corrobora os estudos de Onoyama et al. (2012) e de Loyarte et al. (2015), já que o foco é o planejamento da pesquisa, sendo importante elencar os resultados a serem alcançados, bem como as atividades e recursos necessários. Assim, o modelo conceitual serviu como guia para a construção de um roadmap que possibilitou a visualização da integração entre as principais e potenciais pragas e doenças com as ações de pesquisa, resultados tecnológicos e recursos necessários para a execução das atividades. A representação gráfica foi projetada para um horizonte de 10 anos, durante os anos de 2015 a 2025, tendo em mente ações de curto (3 anos), médio (6 anos) e longo prazo (10 anos).

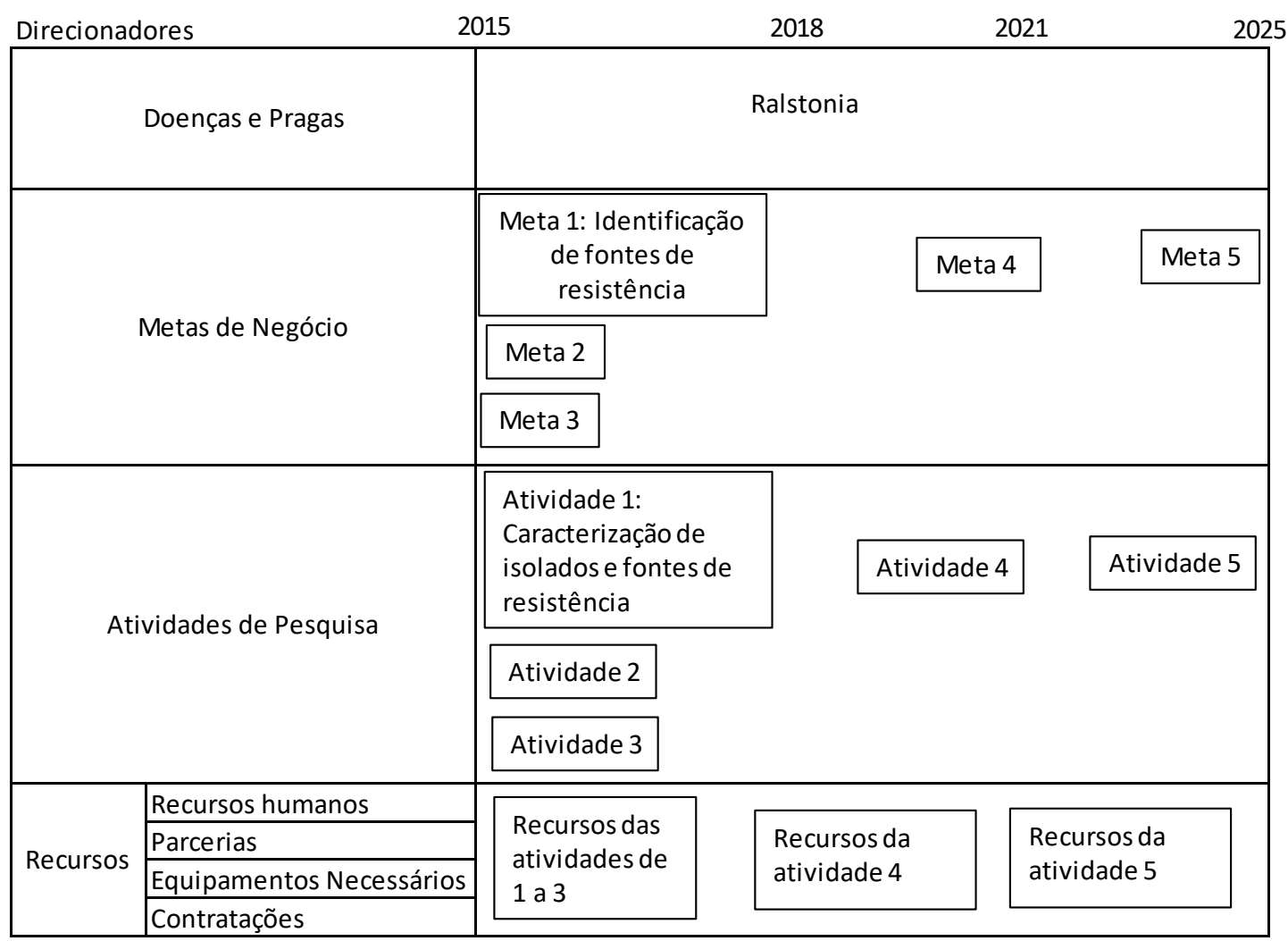

Figura 2 - Roadmap da cadeia de tomate Fonte: Dados da pesquisa. Elaborado pelos autores. 
Na primeira camada, foram elencadas 27 doenças e pragas que afetam ou podem vir a afetar a cultura do tomateiro de mesa, sendo essas divididas da seguinte forma: 7 bactérias, 9 fungos, 6 insetos, 3 nematóides e 5 vírus. As doenças e pragas foram dispostas em ordem de importância, segundo a visão dos pesquisadores da área de tomate. O grupo optou por trabalhar com todas elas, colocando maior ênfase naquelas com maior impacto negativo real, no caso daquelas já trabalhadas, e potencial, no caso daquelas que poderão se tornar problema.

Para cada doença ou praga, foram vinculadas metas de negócio. Para a qualificação das metas dentro da estrutura de projetos da Embrapa, essas foram definidas, considerando os indicadores de resultados adotados pela empresa. Dentre os indicadores de resultados existentes, para este trabalho, foram considerados o avanço do conhecimento científico, novas cultivares, metodologias, práticas e processos agropecuários. Ao todo, foram elencadas 100 metas, distribuídas em curto, médio e longo prazo. Para cada meta, foi vinculada uma atividade, totalizando também 100 (Figura 3). As metas de negócio e atividades de pesquisa das 3 principais bactérias, 3 principais fungos, 3 principais insetos, 3 nematóides e 3 principais vírus corresponderam a $69 \%$ do total.

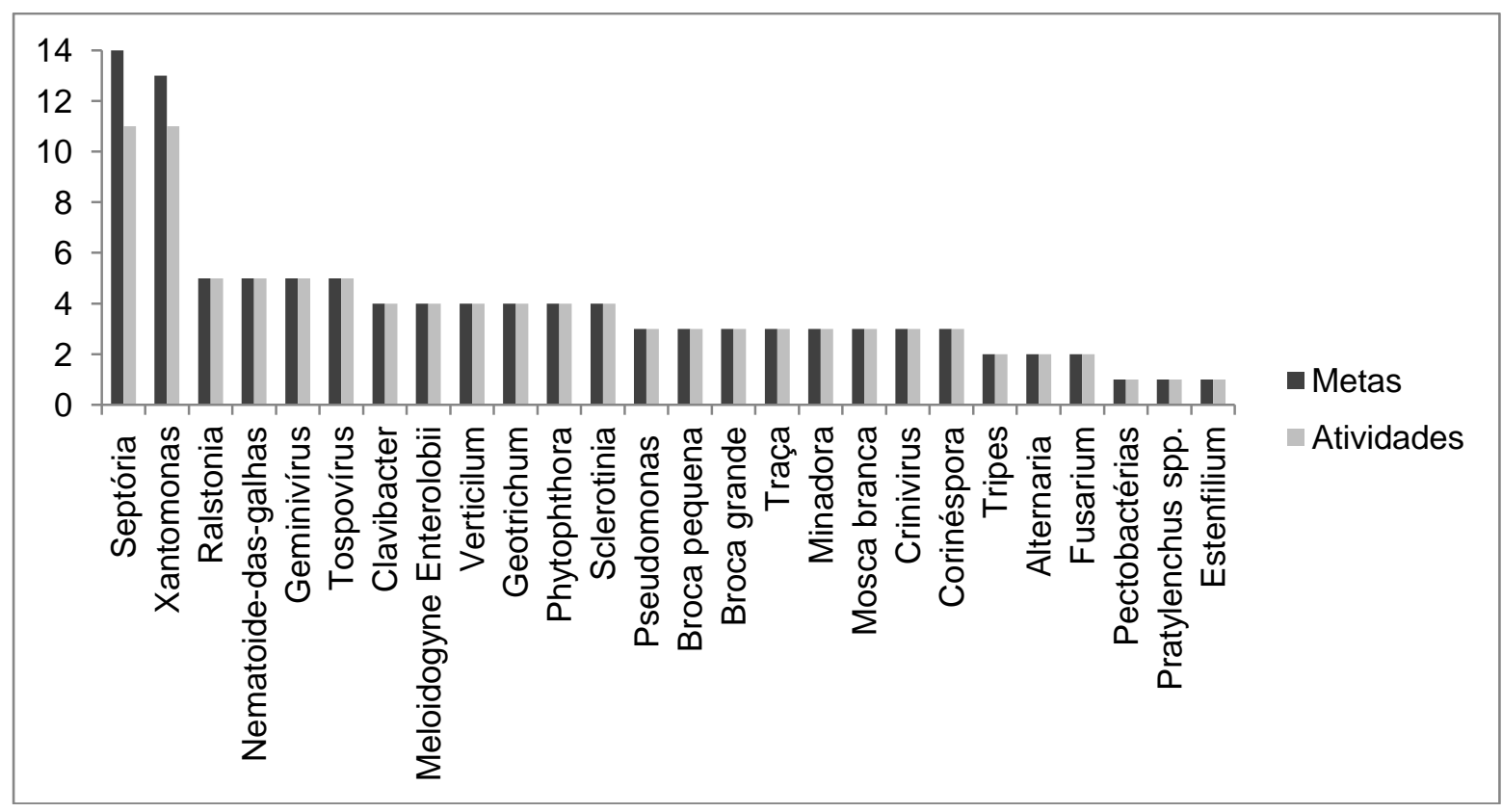

Figura 3 - Distribuição das metas e atividades de pesquisa Fonte: Elaborado pelos autores 
Algumas atividades importantes, correspondendo $2 \%$ do total, foram consideradas de natureza contínua, uma vez que sempre existirá a necessidade de serem realizadas, independentemente dos projetos em execução, como, por exemplo, o monitoramento e desenvolvimento de tecnologias que produzam cultivares mais adaptadas à doenças e pragas ou que permitam a produção em condições desfavoráveis. Outrossim, essas atividades de pesquisa em alguns tipos de doenças e pragas já estão incorporadas aos programas de melhoramento e de fitossanidade, uma vez que existe o risco permanente de quebra da resistência das cultivares de tomate, havendo pouca ação atrelada.

Para melhor ajuste dentro da estrutura de pesquisa da Embrapa, optou-se pelo agrupamento das demais atividades em 3 grandes projetos de pesquisa que envolvem os temas fitossanidade e melhoramento: projeto de curto prazo, projeto de médio prazo e projeto de longo prazo. $47 \%$ das atividades foram vinculadas ao projeto de curto prazo, $34 \%$ das atividades contemplaram o projeto de médio prazo e $13 \%$ as de longo prazo. Há algumas atividades, $3 \%$ do total, que sombreiam as atividades entre curto e médio prazo.

Por fim, a quarta camada abarca os recursos físicos (equipamentos) e humanos (parcerias e contratações), imprescindíveis para viabilizar as ações de pesquisa. Assim como na terceira camada, são listados os recursos já existentes e os futuros. A partir dessas informações, foram identificados os gargalos em termos de contratação de pessoas e novos equipamentos para atender a todas as metas e atividades listadas.

Com essas informações, elaborou-se um plano de ação para os próximos 10 anos, conforme ilustrado na Tabela 1, elencando-se as linhas de pesquisa agrupadas por projetos e relacionadas com as metas a serem alcançadas. Os projetos a serem executados englobam ações de manejo de doenças e pragas, póscolheita e melhoramento de tomate de mesa. 
Tabela 1

Plano de ação

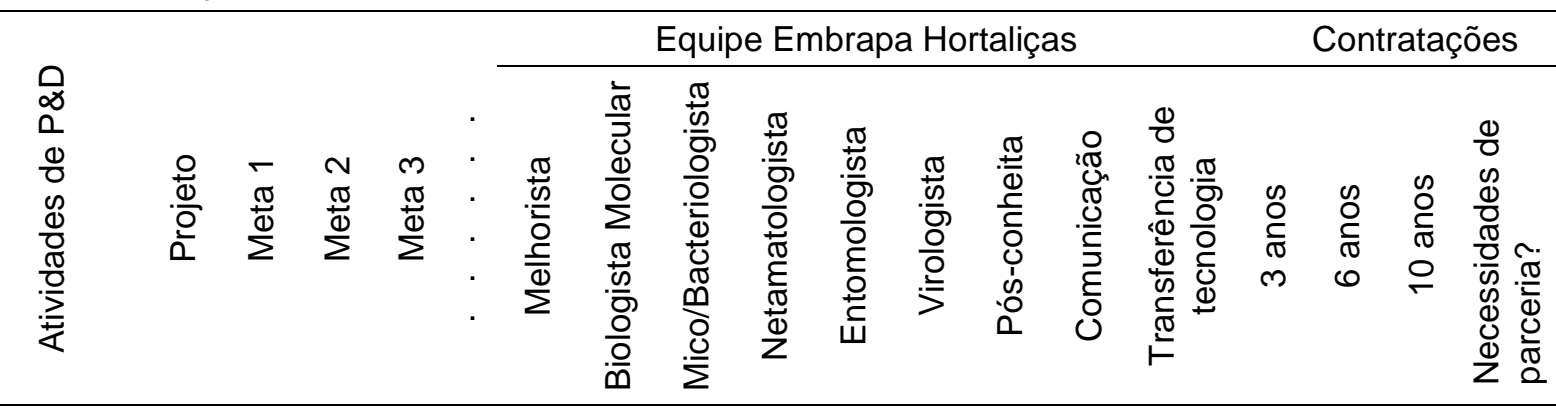

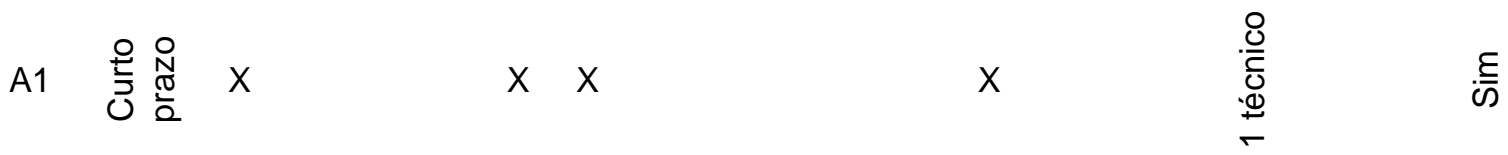

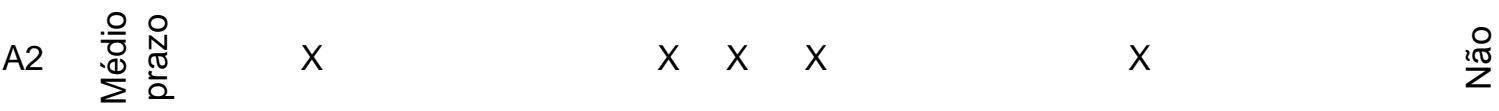

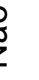

A3

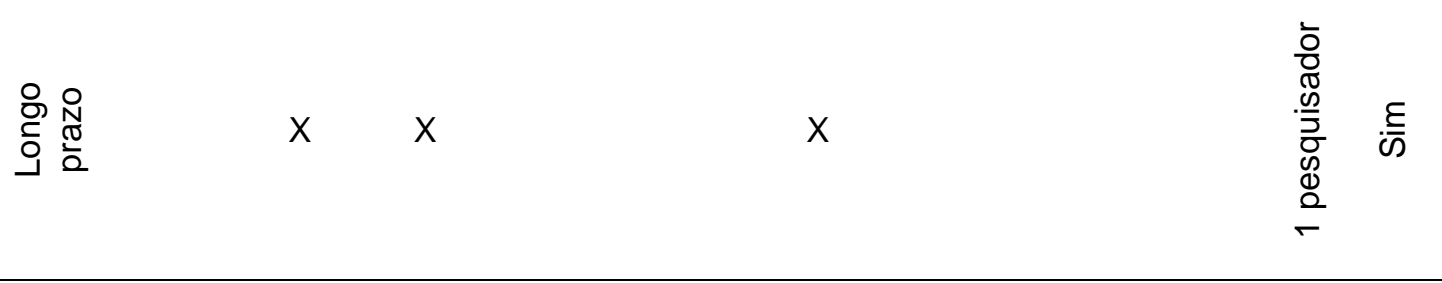

Fonte: Elaborado pelos autores

Legenda-A: atividade

Para cada atividade, foram vinculados tempo de ação (curto, médio ou longo prazo), meta, pesquisadores envolvidos, possíveis necessidades de contratações e levantamento de parcerias existentes e potenciais. As parcerias contribuem para agilização das ações de pesquisa ao possibilitar o uso, de forma coordenada, de competências externas. A representação do plano de ação permitiu visualizar a integração multifuncional entre as áreas de pesquisa da Embrapa Hortaliças envolvidas na execução das ações propostas.

\section{Considerações Finais}

O Technology Roadmapping tem sido aplicado no setor industrial, mostrando grandes benefícios em termos estratégicos para atendimento de mercado e posicionamento de produtos (Carvalho et al., 2013; Phaal et al., 2004; Probert \& Radnor, 2003). No presente estudo, a sua utilização possibilitou visualizar possíveis problemas futuros, permitindo um redirecionamento e abertura de novas linhas de 
pesquisa de forma a antecipar os problemas e minimizar ou eliminar os riscos existentes. Portanto, do ponto de vista acadêmico, os resultados comprovam a versatilidade do uso do referido método, corroborando os achados de Lee e Park (2005) e de Oliveira et al. (2012).

Do ponto de vista gerencial, o estudo permitiu à equipe o ganho de competências na área prospectiva e de planejamento, além de fomentar um espaço de discussão com objetivos mais claros e mais bem direcionados, contando com a participação de profissionais de diversas áreas da organização. Com isso, houve melhora no processo de comunicação dos atores envolvidos em relação à programação de P\&D, em consonância com os estudos de Groenveld (2007), Grossman (2004) e Phaal e Muller (2009). Nesse sentido, essa iniciativa apoia e serve como incentivo para a adoção de novos métodos no processo de planejamento tecnológico da Embrapa, uma vez que o resultado dessa intervenção será incorporado aos projetos atuais e futuros que dizem respeito a doenças e pragas do tomateiro e ao melhoramento de novas cultivares de tomate.

Como limitação, aponta-se que a aplicação e a operacionalização do método foram baseadas em uma demanda da cadeia de tomate de mesa, restringindo a generalização da estrutura apresentada. Ressalta-se a previsão, para estudos futuros, do uso do TRM para o aprimoramento da pesquisa em outras cadeias agrícolas, alavancando a orientação sistemática para 0 processo de desenvolvimento tecnológico do setor agropecuário.

\section{Referências}

Alves, L. B., Silva, C. E. S. D., \& Mello, C. H. P. (2011). Analysis of the use of technology roadmapping as a means of selecting a reference product for reverse engineering. Gestão \& Produção, 18(1), 55-72.

Bassi, N. S., Silva, C. L. D., leis, F., \& Poit, D. R. (2015). O uso de estudos prospectivos na elaboração do planejamento estratégico de uma instituição científico-tecnológica brasileira. Parcerias Estratégicas, 18(37), 173-192.

Carvalho, M. M., Fleury, A., \& Lopes, A. P. (2013). An overview of the literature on technology roadmapping (TRM): Contributions and trends. Technological Forecasting and Social Change, 80(7), 1418-1437.

Coelho, G. M., Santos, D. M. D., Santos, M. D. M., \& Fellows Filho, L. (2010). Caminhos para o desenvolvimento em prospecção tecnológica: Technology 
Roadmapping-um olhar sobre formatos e processos. Parcerias Estratégicas, 10(21), 199-234.

Eden, C., \& Huxham, C. (2001). Pesquisa-ação no estudo das organizações. Handbook de Estudos Organizacionais, 2, 93-117.

Empresa Brasileira de Pesquisa Agropecuária (2015). V Plano-Diretor da Embrapa: 2014-2034. Brasília, DF. 24p.

Food and Agriculture Organization of the United Nations (2014). Food and agriculture data. Recuperado de http://www.fao.org/faostat/en/\#data/QC

Groenveld, P. (2007). Roadmapping integrates business and technology. ResearchTechnology Management, 50(6), 49-58.

Grossman, D. S. (2004). Putting technology on the road. Research-Technology Management, 47(2), 41-46.

Hortifruti Brasil. (2016). Custos sobem ano a ano, cenário não diferente em 2016. Recuperado de http://www.hfbrasil.org.br/br/custo-para-se-produzir-1-hectare-detomate-ultrapassa-r-100-mil.aspx

Huang, L., Zhang, Y., Guo, Y., Zhu, D., \& Porter, A. L. (2014). Fourdimensional Science and Technology planning: A new approach based on bibliometrics and technology roadmapping. Technological Forecasting and Social Change, 81, 3948.

Instituto Brasileiro de Geografia e Estatística. (2015). Levantamento sistemático da produção agrícola. Recuperado de http://www.ibge.gov.br/home/estatistica/indicadores/agropecuaria/lspa/lspa 2016 $\underline{06}$ 5.shtm

Instituto Brasileiro de Geografia e Estatística. (2017). Levantamento sistemático da produção agrícola. Recuperado de http://ftp.ibge.gov.br/Producao Agricola/Levantamento Sistematico da Produca o Agricola [mensal]/Fasciculo/lspa 201612.pdf

Kappel, T. A. (2001). Perspectives on roadmaps: how organizations talk about the future. Journal of Product Innovation Management, 18(1), 39-50.

Kerr, C., \& Phaal, R. (2015). Visualizing roadmaps: A design-driven approach. Research-Technology Management, 58(4), 45-54.

Lee, J. H., Phaal, R., \& Lee, C. (2011). An empirical analysis of the determinants of technology roadmap utilization. $R \& D$ Management, 41(5), 485-508.

Lee, S., \& Park, Y. (2005). Customization of technology roadmaps according to roadmapping purposes: Overall process and detailed modules. Technological Forecasting and Social Change, 72(5), 567-583. 
Loyarte, E., Posada, J., Gaines, S., Rajasekharan, S., Olaizola, I. G., O. Otaegui, \& et al. (2015). Technology roadmapping (TRM) and strategic alignment for an applied research centre: a case study with methodological contributions. $R \& D$ Management, 45(5), 474-486.

Marques, D. V., Vedovoto, G. L., \& Avila, A. (2009). Avaliação de impactos econômicos, sociais e ambientais de tecnologias: a experiência da Embrapa no período. XLI Simpósio Brasileiro de Pesquisa Operacional. Porto Seguro, 15p.

Martins, G. A., \& Theóphilo, C. R. (2009). Metodologia da investigação científica para ciências sociais aplicadas. Atlas.

Melo Filho, L. D. R., Gonçalves, C. A., Cheng, L. C., \& Muniz, R. M. (2015). Abordagem Estratégica de Roadmapping na Geração de Diretrizes de Inovação para Firmas de um Conglomerado Industrial. Revista Ibero-Americana de Estratégia, 14(3), 49.

Natalense, J., \& Zouain, D. (2013). Technology roadmapping for renewable fuels: case of biobutanol in Brazil. Journal of technology management \& innovation, 8(4), 143-152.

Oliveira, M. G., \& Rozenfeld, H. (2010). Integrating technology roadmapping and portfolio management at the front-end of new product development. Technological forecasting and social change, 77(8), 1339-1354.

Oliveira, M. G., Freitas, J. S., Fleury, A. L., Rozenfeld, H., Phaal, R., Probert, \& et al. (2012). Roadmapping: uma abordagem estratégica para o gerenciamento da inovação em produtos, serviços e tecnologias. Rio de Janeiro: Elservier.

Onoyama, S. S., Silva, G. O. D., Cota Júnior, M. B., Cheng, L. C., Lopes, C. A., Vieira, et al. (2012). Technology roadmapping, an alternative for designing agricultural research and its application on the carrot chain. Horticultura Brasileira, 30(4), 572-578.

Phaal, R., Farrukh, C., Mitchell, R., \& Probert, D. (2001). T-Plan: Fast Start to Technology Roadmapping Planning Your Route to Success. University of Cambridge.

Phaal, R., Farrukh, C., Mitchell, R., \& Probert, D. (2003). Starting-up roadmapping fast. Research-Technology Management, 46(2), 52-59.

Phaal, R., Farrukh, C. J., \& Probert, D. R. (2004). Technology roadmapping-a planning framework for evolution and revolution. Technological forecasting and social change, 71(1), 5-26. 
Phaal, R., Farrukh, C. J., \& Probert, D. R. (2005). Developing a technology roadmapping system. Technology Management: A Unifying Discipline for Melting the Boundaries, 31, 99-111.

Phaal, R., Farrukh, C. J., \& Probert, D. R. (2010). Roadmapping for strategy and innovation: aligning technology and markets in a dynamic world. Institute for Manufacturing.

Phaal, R., \& Muller, G. (2009). An architectural framework for roadmapping: Towards visual strategy. Technological Forecasting and Social Change, 76(1), 39-49.

Probert, D., \& Radnor, M. (2003). Frontier experiences from industry-academia consortia. Research-Technology Management, 46(2), 27-30.

Schuh, G., Aghassi, S., Orilski, S., Schubert, J., Bambach, M., Freudenberg, R., \& et al. (2011). Technology roadmapping for the production in high-wage countries. Production Engineering, 5(4), 463-473.

Subramaniam, M., \& Youndt, M. A. (2005). The influence of intellectual capital on the types of innovative capabilities. Academy of Management journal, 48(3), 450463.

Thiollent, M. (2009). Pesquisa-ação nas organizações. Atlas. 